\title{
Optimal control of wind farm power extraction in large eddy simulations
}

\author{
Jay P. Goit* and Johan Meyers ${ }^{\dagger}$ \\ Department of Mechanical Engineering, KU Leuven, \\ Celestijnenlaan 300 bus 2421, B3001 Leuven, Belgium
}

\begin{abstract}
In the present work we couple flow simulations performed using Large Eddy Simulations (LES) with gradient based optimization to control individual turbine in a farm, so as to achieve an increase in the total power output. The controls in our optimization problem are the disk-based thrust coefficients $C_{\mathrm{T}, \mathrm{n}}^{\prime}$ of individual turbines as function of time. We use a gradient-based algorithm for the optimization and the gradients are computed using the adjoint method; the adjoint equations are formulated directly from the LES equation and the cost functional. We employ a receding-horizon predictive control setting and solve the optimization problem iteratively at each time horizon based on the gradient information obtained from the evolution of the flow field and the adjoint computation. In this paper we further elaborate the optimization techniques, interpret the simulation of adjoint field and present results for the wind-farm boundary layer cases. We find that the extracted farm power increases by approximately $20 \%$, during optimal control. However, the increased power output is also responsible for an increase in turbulent dissipation, and a deceleration of the boundary layer. These issues are further discussed.
\end{abstract}

\section{Nomenclature}

$C_{\mathrm{T}, \mathrm{n}}^{\prime}$ Thrust coefficient of $n^{\text {th }}$ turbine

f Wind turbine forces

$\mathbf{f}^{*} \quad$ Adjoint force term

$\tilde{\boldsymbol{u}}, \tilde{\boldsymbol{\xi}}$ Velocity field, adjoint velocity

$\tilde{p}, \tilde{\pi}$ Pressure field, adjoint pressure field

$\bar{V}, \bar{\chi}$ Time and disk averaged velocity at the turbine, its adjoint counterpart

$u_{*} \quad$ Friction velocity

$\mathscr{J}$ Cost functional

L Lagrangian

$\alpha \quad$ step length

\section{Introduction}

In large wind farms, the effect of wakes, and their interaction leads to a reduction in farm efficiency, with power generated by turbines in a farm being lower than that of a lone-standing turbine. Such wake interaction, as well as interaction between a large wind farm and the Atmospheric Boundary Layer (ABL) has received significant attention, recently (see Ref. 1-3). As the understanding of wind farm aerodynamics broadens, our interest is shifting towards exploring the possibilities of optimizing and improving the powerextraction in a farm. One way to achieve the increase in the farm power is by optimizing the wind farm layout, i.e., the number and position of turbines. Next to that, performance of wind farm may also be improved by a coordinated control of all turbine with the aim to increase the overall energy extraction by

*Email: jay.goit@mech.kuleuven.be

†Email: johan.meyers@mech.kuleuven.be 
the farm. In the current work, we focuss on this second aspect, combining optimal control with wind-farm large-eddy simulations.

One of the first studies on wind farm performance was conducted by Newman, ${ }^{4}$ who theoretically determined the effect of spacing on the power output of wind turbines in a large arrays. More recently, Meyers \& Meneveau, ${ }^{5}$ have predicted an optimal turbine spacing as a function of wind farm induced surface roughness as well as in terms of the ratio of turbine costs to land surface costs. While there have been a significant number of studies on the wind turbine control, most of them have focused on the enhancement of the individual turbine performance. Such control, if implemented in a large wind farm can only lead to a suboptimal condition, as it does not account for the interaction between turbines. However, recent trend is switching gradually towards a centralized controller which would optimize the total power output and even minimize the load. An example of considering overall power and load in the wind farm control is presented by Soleimanzadeh et al. ${ }^{6}$ An example of coordinated control was also considered by by Fleming et al. ${ }^{7}$ in their multi-turbine control algorithm to maximize the total power output and reduce the load experienced by the turbines. They analyzed yaw alignment control for two turbine system and found that the use of the controller increased the global power. The studies mentioned above and other work in the literature rely heavily on the simplified model for the turbulent simulation and the wind farm dynamics. These methods have several advantages, such as they are easy to implement and at the same time cheaper and faster to compute; in some cases resulting problem can even be solved analytically. However, they cannot provide a complete picture on the performance of optimization for complex time varying problems such as turbulent flow.

In the present study we couple flow simulation performed using the Large Eddy Simulations (LES) with a gradient based optimization to control individual turbine in a farm, so as to achieve an increase in the total power. The gradients for the optimization algorithm are computed using adjoint-based technique which requires additional simulation of the adjoint field. Adjoint equations are formulated directly from the LES equation and the cost functional. It is perhaps not very surprising that such algorithm is computationally demanding and therefore prohibitive for implementing directly as an online controller in a real wind farm. However, the results obtained with this approach allow us to identify the best possible performance of the controls for the turbines in the given farm layout and flow condition. This can act as a reference for future investigation into more practical models.

In this paper we present the development of wind farm power optimization technique in the frame work of the LES simulation. To this end, we employ a receding-horizon predictive control setting and solve the optimization problem iteratively at each time horizon based on the gradient information obtained from the evolution of flow field and adjoint computation. A similar strategy is used by Bewley et al. ${ }^{8}$ in their study on the reduction of turbulent kinetic energy and drag of a turbulent channel flow. We further discuss the various factors and constraints that should be taken into account to achieve the power gain in a large scale farm.

The paper is further organized as follows. We begin by presenting our problem of interest, in particular, the cost functional and the governing flow equations in section II. Section III provides the formal derivation of the set of adjoint equations, followed by the description of the numerical method and case setup in section IV. Results for the uncontrolled simulation and optimization case are discussed subsequently in section V and section VI respectively. Finally, in section VII main conclusions and future research directions are presented.

\section{Problem formulation}

In the present paper we consider the optimization of a wind farm in a fully developed turbulent boundary layer, with the flow field information computed using LES. For the sake of convenience, the three-dimensional flow domain $\Omega$, and the boundaries $\Gamma$ are illustrated in figure 1 . The governing equations are the filtered incompressible Navier-Stokes (NS) and continuity equations,

$$
\begin{gathered}
\frac{\partial \tilde{\boldsymbol{u}}}{\partial t}+\tilde{\boldsymbol{u}} \cdot \nabla \tilde{\boldsymbol{u}}=-\frac{1}{\rho} \nabla \tilde{p}-\nabla \cdot \tau_{\text {sgs }}+\mathbf{f}, \\
\nabla \cdot \tilde{\boldsymbol{u}}=0,
\end{gathered}
$$

where $\tilde{\boldsymbol{u}}$ is the filtered velocity field, $\tilde{p}$ the pressure, $\tau_{s g s}$ the subgrid-scale tensor and $\mathbf{f}$ the forcing term due to turbines. This turbine induced force is based on the classical actuator-disk method which models the 


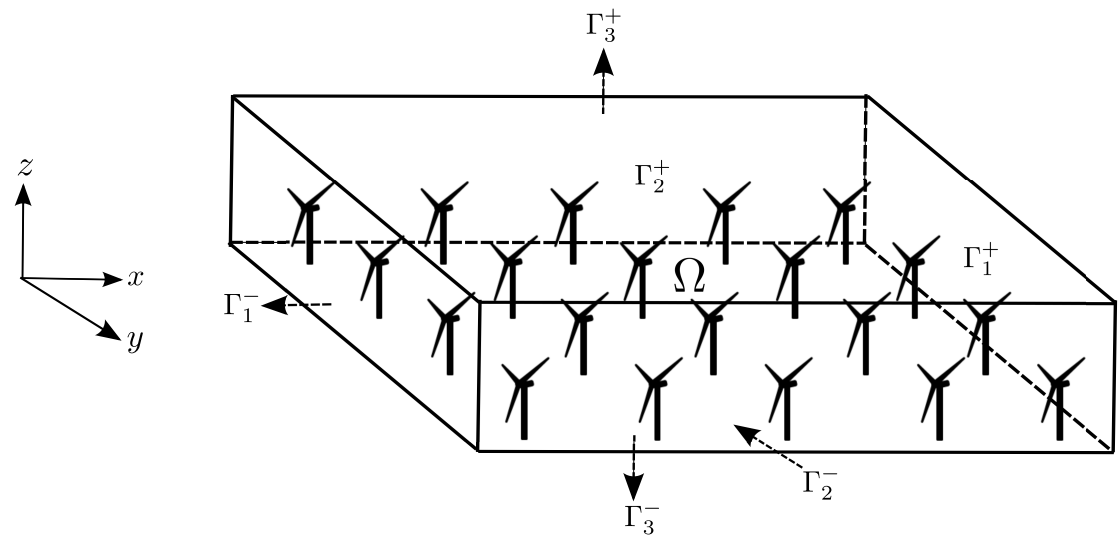

Figure 1. Flow region. Domain is denoted by $\Omega$ and boundaries are denoted by $\Gamma$

total thrust force acting on fluid due to the turbine and is written as, ${ }^{9}$

$$
f_{\mathrm{n}}=-\frac{1}{2} C_{\mathrm{T}, \mathrm{n}}^{\prime} \bar{V}_{\mathrm{n}}^{2} \mathscr{R}_{1, \mathrm{n}}(\mathbf{x}),
$$

where $\mathrm{n}=1,2, \ldots, N_{t}$ and $N_{t}$ is the total number of turbines, $C_{\mathrm{T}, \mathrm{n}}^{\prime}$ is a thrust coefficient defined using the velocity at the turbine-disk. The turbine force is first defined on a coordinate system in a turbine rotor plane with a fine mesh and then projected smoothly on to the coarser LES grid using a Gaussian convolution filter given by

$$
\mathscr{R}_{1, \mathrm{n}}(\mathbf{x})=\iiint G\left(\mathbf{x}-\mathbf{x}^{\prime}\right) \delta\left(\mathbf{e}_{\perp} \cdot\left(\mathbf{x}^{\prime}-\mathbf{x}_{\mathbf{0}}\right)\right) \mathrm{H}\left(R-\left\|\mathbf{y}\left(\mathbf{x}^{\prime}-\mathbf{x}_{\mathbf{0}}\right)\right\|\right) \mathrm{d}^{3} \mathbf{x}^{\prime} .
$$

In order to determine the disk-averaged local velocity $\bar{V}_{\mathrm{n}}$, first spatial averaging is performed using the geometrical rotor footprint $\mathscr{R}_{1, \mathrm{n}}(\mathbf{x})$ as a weighing factor

$$
V_{\mathrm{n}}(t)=\frac{1}{\pi r_{\mathrm{n}}^{2}} \iiint \mathscr{R}_{1, \mathrm{n}}(\mathbf{x}) \tilde{\boldsymbol{u}}(x) \cdot \mathbf{e}_{\perp} \mathrm{d}^{3} \mathbf{x}
$$

where $r_{\mathrm{n}}$ is the radius of the rotor, and $\tilde{\boldsymbol{u}}(x)$ is the local instantaneous velocity. This operation is followed by a local time average that is performed using exponential time filter given by

$$
\sigma \frac{\mathrm{d} \bar{V}_{\mathrm{n}}}{\mathrm{d} t}=\left(V_{\mathrm{n}}-\bar{V}_{\mathrm{n}}\right)
$$

with $\sigma$ a time window that we take equal to 5 seconds in the current work. More details about the actuator disk implementation can be found in Ref. 1 and 9.

Finally, as in any other optimization problem, it is necessary to identify and define a cost functional appropriate for the problem of interest. It must be clear from the text above that any relevant cost functional for this problem should contain total farm power. Therefore, we chose the cost functional of the form

$$
\begin{aligned}
\mathscr{J} & =\int_{0}^{T} \sum_{n=1}^{N_{t}}-P_{\mathrm{n}} d t \\
& =\int_{0}^{T} \int_{\Omega} \sum_{n=1}^{N_{t}}-\frac{1}{2} C_{\mathrm{T}, \mathrm{n}}^{\prime} \bar{V}_{\mathrm{n}}^{2} V_{\mathrm{n}}(t) \mathscr{R}_{1, \mathrm{n}}(\mathbf{x}) d \Omega d t,
\end{aligned}
$$

where $P_{\mathrm{n}}$ is the power extracted by turbine $\mathrm{n}\left(\mathrm{n}=1 \cdots N_{t}\right)$. The control parameters are the turbine thrust coefficients $C_{\mathrm{T}, \mathrm{n}}^{\prime}$. When the optimization is performed, $C_{\mathrm{T}, \mathrm{n}}^{\prime}$ will change from turbine to turbine and also vary in time, it is a function of turbine and time. In a physical sense $C_{\mathrm{T}, \mathrm{n}}^{\prime}$ can be considered to be a parameter that represents the effects of changing the blade pitch of the turbines either capturing more or less of the available energy in the wind. In the current work, we do not represent the blade pitch directly in our control model. 


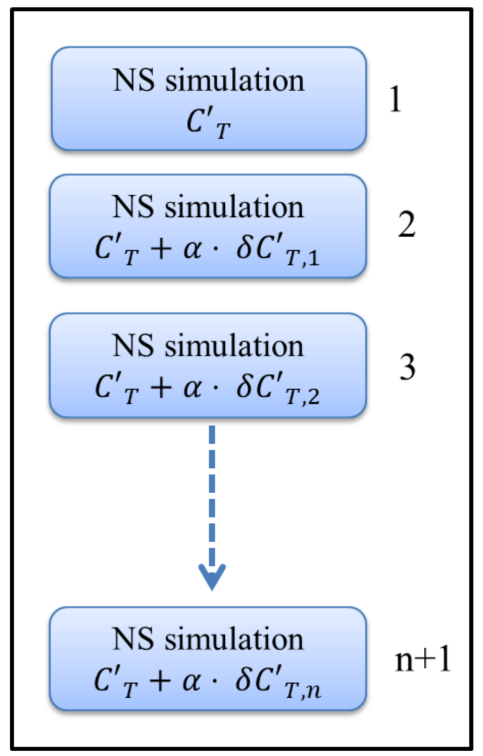

(a)

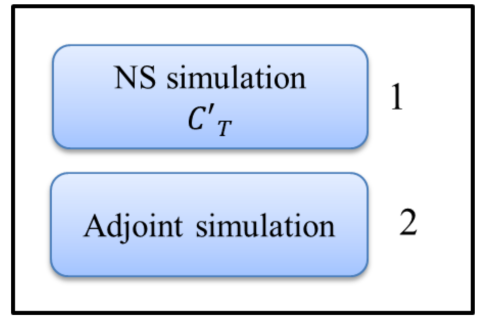

(b)

Figure 2. Methods for the evaluation of gradients. (a) Classical method, (b) Adjoint based method.

Based on the above discussions regarding the cost functional and the governing flow equations, we can finally construct our optimization problem as:

$$
\begin{array}{ll}
\min \mathscr{J}=\int_{0}^{T} \int_{\Omega} \sum_{n=1}^{N_{t}}-\frac{1}{2} C_{\mathrm{T}, \mathrm{n}}^{\prime} \bar{V}_{\mathrm{n}}^{2} V_{\mathrm{n}}(t) \mathscr{R}_{1, \mathrm{n}}(\mathbf{x}) d \Omega d t & \\
\text { s.t. } & \\
\frac{\partial \tilde{\boldsymbol{u}}}{\partial t}+\tilde{\boldsymbol{u}} \cdot \nabla \tilde{\boldsymbol{u}}=-\frac{1}{\rho} \nabla \tilde{p}-\nabla \cdot \tau_{\text {sgs }}+\mathbf{f} & \text { in } \mathbf{Q}:=\Omega \times(0, T] \\
\nabla \cdot \tilde{\boldsymbol{u}}=0 & \text { in } \mathbf{Q}:=\Omega \times(0, T] \\
\frac{d \tilde{u}_{1}}{d z}=0, \frac{d \tilde{u}_{2}}{d z}=0, \tilde{u}_{3}=0 & \text { on } \Gamma_{3}^{+} \times(0, T] \\
\tau_{w, i}=-\left[\frac{\kappa}{\log \left(z / z_{0}\right)}\right]^{2}\left(\overline{\tilde{u}}_{1}^{2}+\overline{\tilde{u}}_{2}^{2}\right)^{1 / 2} \overline{\tilde{u}}_{i}(i=1,2) & \text { on } \Gamma_{3}^{-} \times(0, T] \\
\frac{\mathrm{d} \bar{V}_{\mathrm{n}}}{\mathrm{d} t}=\frac{1}{\sigma}\left(V_{\mathrm{n}}-\bar{V}_{\mathrm{n}}\right) & \text { in }(0, T]
\end{array}
$$

Note that expressions for the top and bottom surface boundary conditions are also added to Eq. (8). At the top of the domain $\left(\right.$ on $\Gamma_{3}^{+}$), a symmetry condition with zero vertical velocity and zero shear stress is used. The boundary condition imposed at the bottom surface comes from relating wall stress $\tau_{w, i}$ to the velocity at the first grid-point using Monin-Obukhov similarity theory. ${ }^{10}$ The boundary conditions in the horizontal directions are periodic, because of the pseudo-spectral discretization techniques used in these directions. The flow is driven by a constant pressure gradient in the $x$ direction, that is implemented using a constant forcing term.

\section{Adjoint formulation for LES of wind farms}

The present section briefly explains the derivation of adjoint equations which should be solved for the gradient evaluation. The adjoint method has long been established as a preferable choice in the gradientbased optimization problem when the number of control variables is large. Since early days, it has, e.g., 
found its application to problem in fluid dynamic design. ${ }^{11,12}$ The strong point of this approach is that the computation of the complete gradient is realized from one forward and one adjoint simulation. Therefore, as schematically shown in figure 2 , it has a large advantage over a conventional forward method, especially when the number of control parameters is very large. For instance, using a forward method (cf. figure 2a) to evaluate gradient in the current setting of a large wind farm, requires one flow simulation for each control parameter totalling to approximately 20,000 large-eddy simulations for the gradient. The same can be achieved using just two simulation, i.e. one forward and one adjoint simulation. The computational cost and time for the adjoint simulation is approximately same as the corresponding forward flow simulation.

We employ a formal Lagrangian method for the derivation of adjoint equations. A similar method is presented by Choi et al. ${ }^{13}$ Readers can also refer to Ref. 14 for the rigorous mathematical proofs and discussions regarding the necessary optimality conditions and other issues related to optimization of partial differential equations.

For every state variables $(\tilde{\boldsymbol{u}}, \tilde{p}, \bar{V})$, also known as primal variables, an adjoint variable $(\tilde{\boldsymbol{\xi}}, \tilde{\pi}, \bar{\chi})$ is associated. These adjoint variables also act as the Lagrange multipliers. We associate following Lagrangian function to our optimization problem stated in Eq. (8).

$$
\begin{aligned}
\mathbf{L}(\tilde{\boldsymbol{u}}, \tilde{p}, \bar{V}, \tilde{\boldsymbol{\xi}}, \tilde{\pi}, \bar{\chi}) & =\mathscr{J}+\int_{0}^{T} \int_{\Omega}\left[\frac{\partial \tilde{\boldsymbol{u}}}{\partial t}+\tilde{\boldsymbol{u}} \cdot \nabla \tilde{\boldsymbol{u}}+\frac{1}{\rho} \nabla \tilde{p}+\nabla \cdot \tau_{s g s}-\mathbf{f}-\tau_{w, i} \delta(z)\right] \cdot \tilde{\boldsymbol{\xi}} d \Omega d t \\
& -\int_{0}^{T} \int_{\Omega} \tilde{\pi} \cdot \operatorname{div} \tilde{\boldsymbol{u}} d \Omega d t+\int_{0}^{T} \sum_{n=1}^{N_{t}}\left[\sigma \frac{\mathrm{d} \overline{\mathbf{V}}_{\mathrm{n}}}{\mathrm{d} t}-\left(V_{\mathrm{n}}-\bar{V}_{\mathrm{n}}\right)\right] \cdot \bar{\chi}_{\mathrm{n}} d t
\end{aligned}
$$

For the optimal condition, we expect that the partial derivatives of $\mathbf{L}$ with respect to the state variables $(\tilde{\boldsymbol{u}}$, $\tilde{p}, \bar{V})$ are zero, i.e.,

$$
\begin{aligned}
& L_{\tilde{p}}(\delta \tilde{p})=0, \\
& L_{\tilde{\boldsymbol{u}}}(\delta \tilde{\boldsymbol{u}})=0, \\
& L_{\bar{V}}(\delta \bar{V})=0,
\end{aligned}
$$

where $\delta \tilde{p}, \delta \tilde{\boldsymbol{u}}$ and $\delta \bar{V}$ are the directions in which derivatives are taken. Substituting from the definition of Lagrangian of Eq. (9) and performing integration by parts in Eq. (10) leads to a set of adjoint equations:

$$
\begin{array}{ll}
-\frac{\partial \tilde{\boldsymbol{\xi}}}{\partial t}-(\tilde{\boldsymbol{u}} \cdot \nabla) \tilde{\boldsymbol{\xi}}-(\nabla \tilde{\boldsymbol{\xi}})^{T} \tilde{\boldsymbol{u}}=-\frac{1}{\rho} \nabla \tilde{\pi}-\nabla \cdot \tau_{s g s}^{*}+\mathbf{f}^{*} & \text { in } \mathbf{Q}:=\Omega \times(0, T] \\
\nabla \cdot \tilde{\boldsymbol{\xi}}=0 & \text { in } \mathbf{Q}:=\Omega \times(0, T] \\
\frac{d \tilde{\xi}_{1}}{d z}=0, \frac{d \tilde{\xi}_{2}}{d z}=0, \tilde{\xi}_{3}=0 & \text { on } \Gamma_{3}^{+} \times(0, T] \\
\tau_{w, i}^{*}=-\left[\frac{\kappa}{\log \left(z / z_{0}\right)}\right]^{2}\left\{\left(\overline{\tilde{u}}_{1}^{2}+\overline{\tilde{u}}_{2}^{2}\right)^{1 / 2} \tilde{\xi}_{i}+\frac{\overline{\tilde{u}}_{1} \overline{\tilde{u}}_{i} \tilde{\xi}_{1}+\overline{\tilde{u}}_{i} \overline{\tilde{u}}_{2} \tilde{\xi}_{2}}{\left.\left(\overline{\tilde{u}}_{1}^{2}+\overline{\tilde{u}}_{2}^{2}\right)^{1 / 2}\right\}}(i=1,2)\right. & \text { on } \Gamma_{3}^{-} \times(0, T] \\
\frac{\mathrm{d} \bar{\chi}_{n}}{\mathrm{~d} t}=\frac{1}{\sigma}\left[\bar{\chi}_{n}+C_{\mathrm{T}, \mathrm{n}}^{\prime} \bar{V}_{n} \int_{\Omega}\left\{-V_{\mathrm{n}}+\tilde{\xi}\right\} \mathscr{R}_{1, n}(\mathbf{x}) d \Omega\right] & \text { in }(0, T]
\end{array}
$$

Comparison of Eq. (11) and Eq. (8) shows that adjoint equations bear a significant similarity to the governing flow equations. Both of them have momentum as well as continuity equations, and similar boundary conditions. However, adjoint equations must be solved backward in time because of the negative sign of the time derivative term. It should also be noted that velocity field information from the forward flow simulation is required in the convective, sub-grid scale and wall-stress terms of the adjoint system and therefore the complete flow field information over the optimization horizon has to be stored to compute the adjoint field. The force term $\mathbf{f}^{*}$ acts as a driving force for adjoints and is primarily decided by the definition of the cost functional. This adjoint force for the current problem is written as

$$
\mathbf{f}^{*}=\sum_{n=1}^{N_{t}} \bar{\chi}_{n} \mathscr{R}_{1, \mathrm{n}}(\mathbf{x})+\sum_{n=1}^{N_{t}} \frac{1}{2} C_{\mathrm{T}, \mathrm{n}}^{\prime} \bar{V}_{\mathrm{n}}^{2} \mathscr{R}_{1, \mathrm{n}}(\mathbf{x}) .
$$


Next let us compare sub-grid scale model for the flow and adjoint formulations. The subgrid-scale model used in this study is a standard Smagorinsky model with a constant coefficient $C_{s}=0.14$, and Mason and Thomson's damping function is used near the wall. ${ }^{15}$ The SGS model for the flow equation is given by

$$
\begin{aligned}
& \tau_{i j}=2 \ell_{s}^{2} \overline{\mathcal{S}} \bar{S}_{i j}, \\
& \bar{S}_{i j}=\frac{1}{2}\left(\frac{\partial \tilde{u}_{i}}{\partial x_{j}}+\frac{\partial \tilde{u}_{j}}{\partial x_{i}}\right), \\
& \overline{\mathcal{S}}=\left(2 \bar{S}_{i j} \bar{S}_{i j}\right)^{\frac{1}{2}},
\end{aligned}
$$

where $\ell_{s}$ is a characteristic length-scale for the eddy-viscosity. Similarly, the SGS in the adjoint equation corresponds to

$$
\begin{aligned}
& \tau *_{i j}=2 \ell_{s}^{2}\left(\overline{\mathcal{S}}^{*} \bar{S}_{i j}+\overline{\mathcal{S}} \bar{S}_{i j}^{*}\right), \\
& \bar{S}_{i j}^{*}=\frac{1}{2}\left(\frac{\partial \tilde{\xi}_{i}}{\partial x_{j}}+\frac{\partial \tilde{\xi}_{j}}{\partial x_{i}}\right), \\
& \overline{\mathcal{S}}^{*}=\frac{\left(2 \bar{S}_{i j} \bar{S}_{i j}^{*}\right)}{\overline{\mathcal{S}}} .
\end{aligned}
$$

We can see that the adjoint SGS is more complicated than the original SGS model in the forward equation. It also uses the filtered rate of strain tensor $\bar{S}_{i j}$ and the characteristic filtered rate of strain $\overline{\mathcal{S}}$ from the flow simulation, which makes this term one of the main challenges in the implementation of adjoint equations.

Finally, let us close this section by defining the gradient of the cost functional. The sensitivity of the cost functional can be computed from the partial derivative of $L$ with respect to the control parameter $C_{\mathrm{T}, \mathrm{n}}^{\prime}$.

$$
\begin{aligned}
\mathbf{L}_{C_{\mathrm{T}, \mathrm{n}}^{\prime}}\left(\delta C_{\mathrm{T}}^{\prime}\right) & =\frac{\partial \mathscr{J}}{\partial C_{\mathrm{T}, \mathrm{n}}^{\prime}}\left(\delta C_{\mathrm{T}}^{\prime}\right)+\int_{0}^{T} \int_{\Omega} \sum_{n=1}^{N_{t}} \frac{1}{2}\left(\delta C_{\mathrm{T}, \mathrm{n}}^{\prime}\right) \bar{V}_{n}^{2} \mathscr{R}_{1, \mathrm{n}}(\mathbf{x}) \tilde{\xi} d \Omega d t \\
& =\int_{0}^{T} \int_{\Omega} \sum_{n=1}^{N_{t}} \frac{1}{2}\left(\delta C_{\mathrm{T}, \mathrm{n}}^{\prime}\right) \bar{V}_{n}^{2}\left\{-V_{n}+\tilde{\xi}\right\} \mathscr{R}_{1, \mathrm{n}}(\mathbf{x}) d \Omega d t
\end{aligned}
$$

Here, $\delta C_{\mathrm{T}}^{\prime}$ is the direction or a small perturbation to the control. As Eq. (15) holds for any arbitrary value of $\delta C_{\mathrm{T}}^{\prime}$, the gradient can easily be identified from the above expression as

$$
\nabla \mathscr{J}=\int_{0}^{T} \int_{\Omega} \sum_{n=1}^{N_{t}} \frac{1}{2} \bar{V}_{n}^{2}\left\{-V_{n}+\tilde{\xi}\right\} \mathscr{R}_{1, \mathrm{n}}(\mathbf{x}) d \Omega d t .
$$

Calculation of the gradient in Eq. (16) is straightforward, $V_{n}$ and $\bar{V}_{n}$ come from the flow simulation and $\tilde{\xi}$ is the solution of adjoint simulation (c.f. Eq. (11)). It should be noted that after discretization, the gradient takes the form of a vector, with dimensions that are equal to that of the control parameter.

\section{Numerical Method}

\section{A. Optimization technique}

A receding-horizon model predictive control technique used in this work is presented schematically in figure 3. A similar setting is employed by Bewley et al. ${ }^{8}$ in the context of drag reduction. In this approach total time $T$ is divided into several optimization horizons of size $T_{\mathrm{H}}$. An optimization horizon is initiated with a constant value for all the control elements, i.e., all the turbines with same $C_{\mathrm{T}}^{\prime}$. With this control and the initial flow field information, a flow simulation (dashed line) followed by an adjoint simulation (red line) is performed to evaluate the cost functional and its gradient. The gradient information so determined is used to update $C_{\mathrm{T}}^{\prime}$ in the direction that reduces the cost function (or increases the power in our case). The updated control is used to compute new flow and gradient information for the next iteration, so that the control can further be modified. This process is repeated for several iterations to achieve a decent locally 


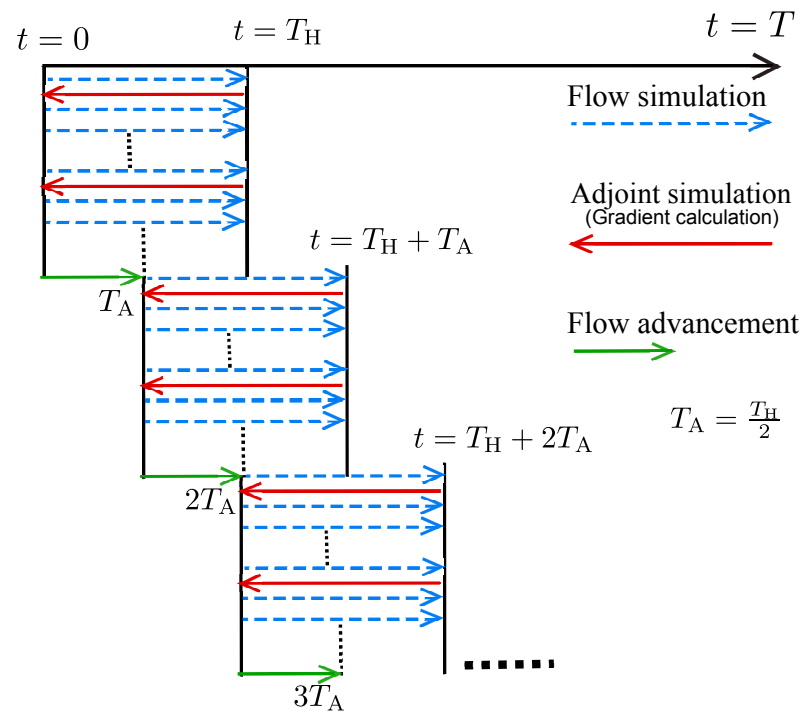

Figure 3. Optimization sequence and advancement.

optimized control and then the simulation is advanced with this new value for the control over the time $T_{\mathrm{A}}$ (green line). Optimization over the next time horizon $\left(T_{\mathrm{A}}, T_{\mathrm{H}}+T_{\mathrm{A}}\right)$ is started anew, repeating all the processes explained above.

The optimization horizon $T_{\mathrm{H}}$ is an important parameter which should be chosen based on various factors. It is obvious that larger $T_{\mathrm{H}}$, the more the objective of the optimization is represented. But at the same time, very large $T_{\mathrm{H}}$ also increases computational challenges, such as storage of the flow field for adjoint simulation, increase in the size of controls etc. One additional constraints in the current frame work is the periodic boundary condition in streamwise direction, which means $T_{\mathrm{H}}$ should be smaller than through-flow time so that the influence of periodicity on the optimization is avoided. We choose $T_{\mathrm{H}}$ to be the time taken by the flow to cross four turbines in a row and flow advancement time $T_{\mathrm{A}}=T_{\mathrm{H}} / 2$. Both these values are pragmatic choices specific to the current problem setting, taking into account all the factors mentioned above.

The control $C_{\mathrm{T}, \mathrm{n}}^{\prime}(t)$ for the particular optimization horizon is improved iteratively using the relation

$$
C_{\mathrm{T}, \mathrm{n}}^{\prime(k+1)}=C_{\mathrm{T}, \mathrm{n}}^{\prime(k)}+\alpha \delta C_{\mathrm{T}, \mathrm{n}}^{\prime(k)},
$$

where $\delta C_{\mathrm{T}, \mathrm{n}}^{\prime(k)}$ is the search direction, $\alpha$ is the step length and $\mathrm{k}$ is the iteration number. The search direction $\delta C_{\mathrm{T}, \mathrm{n}}^{\prime(k)}$ is determined using efficient Polak-Ribiére conjugate-gradient method which depends on the gradient of the cost functional and the search direction from previous step such that

$$
\delta C_{\mathrm{T}}^{\prime(k)}=-\nabla \mathscr{J}^{(k)}+\beta^{k} \delta C_{\mathrm{T}}^{(k-1)},
$$

where the coefficient $\beta^{k}$ is given by

$$
\beta^{k}=\frac{\left(\nabla \mathscr{J}^{(k)}-\nabla \mathscr{J}^{(k-1)}\right) \cdot \nabla \mathscr{J}^{(k)}}{\left(\nabla \mathscr{J}^{(k-1)} \cdot \nabla \mathscr{J}^{(k)}\right)} .
$$

The step length $\alpha$ (c. f. Eq. (17)) governs the size of the update that should be made, so that the new control at each iteration minimizes the cost functional locally. The optimum value for $\alpha$ along the search direction is computed using an iterative line search method based on mnbrak and Brent algorithms. ${ }^{16}$ For details about search direction and line search algorithm used in this study, readers are referred to Delport et al. (Ref. 17,18), who have well documented these techniques and their implementation in their study on the optimal control of turbulent mixing-layer.

\section{B. Discretization and Case setup}

The SP-Wind code used in this study is an in-house code developed in earlier studies. ${ }^{1,5,9}$ The code uses pseudo-spectral discretization techniques with the $3 / 2$ de-aliasing of the convective and SGS terms 


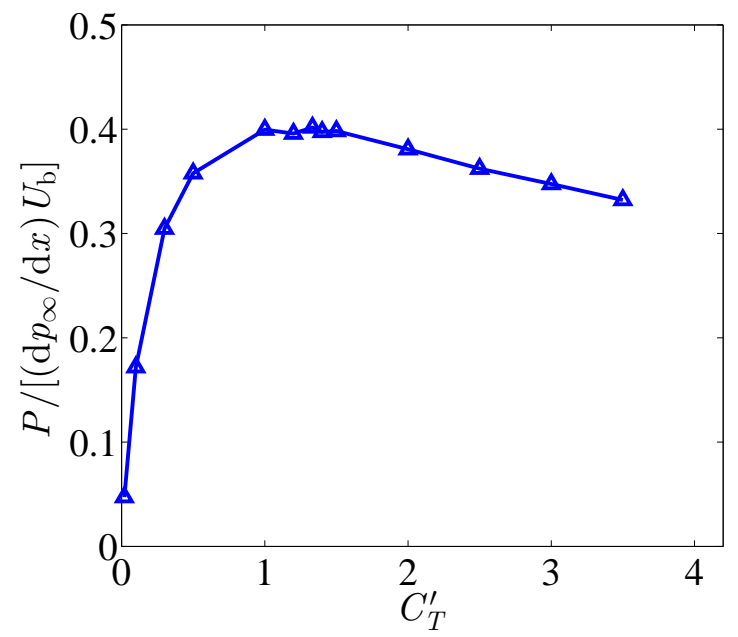

Figure 4. Mean power output of uncontrolled wind farm as function of $C_{\mathrm{T}}^{\prime}$. Power is normalized by the driving power.

in the horizontal directions. In the vertical direction a fourth-order energy-conservative finite difference discretization is used. Time advancement is performed using a four-stage fourth-order Runge-Kutta scheme. The time step is set to a fixed value of 0.7 seconds, which corresponds approximately to a CFL number of 0.4. Boundary conditions, the SGS model as well as the model for the wind turbines have already been explained in Section II.

Discretization of adjoint equation is very similar to that of the flow problem and is solved on the same grid resolution. We use the same pseudo-spectral techniques in the horizontal directions and finite difference discretization in the vertical direction. The velocity field from forward simulation is stored every time step and the same field is used for all four Runge-Kutta stages in the adjoint simulations. Additionally, the adjoint system is solved at the same points in time as its flow counterpart, but backward in time.

Simulations are performed on a mesh of $N_{x} \times N_{y} \times N_{z}=256 \times 192 \times 81$ and a computational domain of size $L_{x} \times L_{y} \times H=7.0 \times 3.0 \times 1\left(\mathrm{~km}^{3}\right)$. The farm consists of $N_{\mathrm{t}}=50$ turbines, in a rectangular arrangement of $10 \times 5$. All 50 turbines are of same size with the rotor diameter $D=100(\mathrm{~m})$ and are assumed to have identical blade geometry as well as performance. The turbines are separated by $S_{\mathrm{x}}=7 D$ and $S_{\mathrm{y}}=6 D$ in $x$ and $y$ directions, respectively. Principal focus in the current study is on turbine optimal control, and therefore turbine spacing is not considered as an optimization parameter, retaining the same wind farm layout throughout the optimization. Turbines with varying rotor diameter or hub height are also excluded from this work.

\section{Power Output of uncontrolled wind farm}

Before going into the discussion about the performance of the controlled wind farm, we treat the power output for uncontrolled simulation cases. For this, simulations are performed for constant $C_{T}^{\prime}$ values for all the turbines in a farm. Layout of the farm is identical to the one used in the later studies, so that these results can act as a reference for controlled simulations.

Figure 4 shows the total farm power averaged over time, for $C_{T}^{\prime}$ values in the range of 0 to 3.5. The farm power is normalized by the driving power due to the pressure-gradient forcing $\left[\left(\mathrm{d} p_{\infty} / \mathrm{d} x\right) U_{\mathrm{b}}\right]$, with $U_{\mathrm{b}}$ being the bulk velocity. As can be seen from figure 4, the farm power initially increases, but after reaching a peak value at $C_{T}^{\prime}=1.33$, it starts to decrease. This behavior is very similar to that of a lone standing turbine; however, for a single turbine case the maximum power, dictated by the Betz limit, is attained at $C_{T}^{\prime}=2.0$.

For an infinite wind farm, it is known that the energy extraction is regulated by the turbulent energy transport down to the farm, ${ }^{1,19}$ and thus the optimal value of $C_{T}^{\prime}=2.0$ is not just regulated by the Betz limit, but also by the farm layout and surface roughness. For the current layout, we find $C_{T}^{\prime}=1.33$ to be the optimum thrust coefficient in an uncontrolled wind farm. We use the power output at this value of $C_{T}^{\prime}$ normalized by $\left[\left(\mathrm{d} p_{\infty} / \mathrm{d} x\right) U_{\mathrm{b}}\right]$ as reference to compare and judge the effectiveness of the optimal control in the controlled simulations. We also prescribe this value as the initial thrust coefficients from which we start 


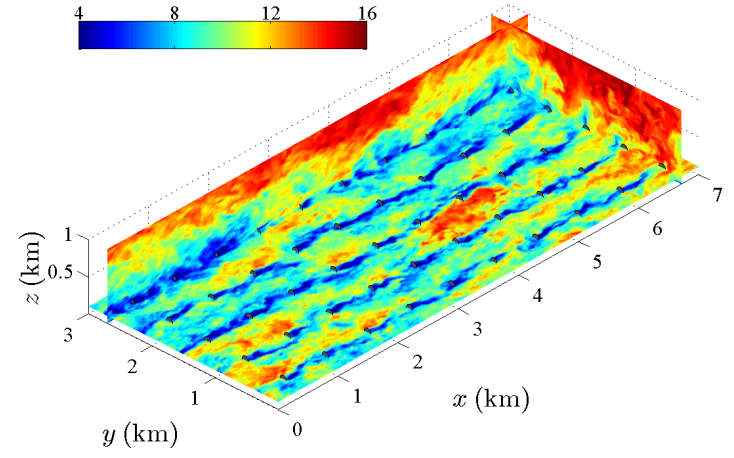

(a)

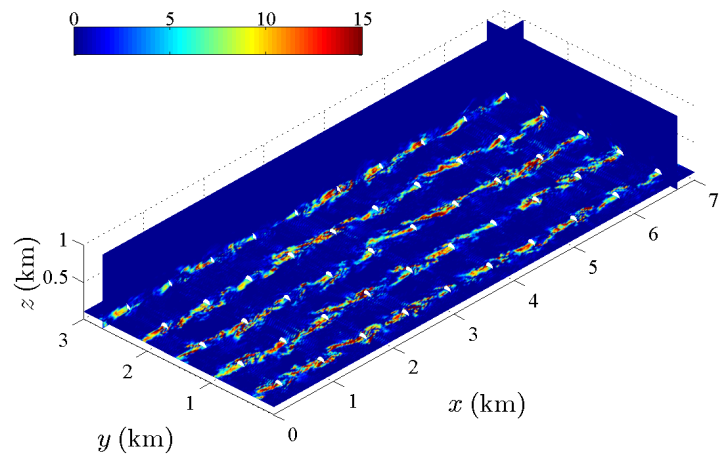

(b)

Figure 5. Contours of instantaneous field. (a) Streamwise velocity field, (b) Streamwise component of adjoint field at time $T_{\mathrm{H}}-t=91$ seconds. Horizontal planes in the figures are taken at the hub height.

the the optimization.

\section{Performance of optimally controlled wind farm}

In figure 5, the instantaneous velocity and adjoint fields are shown. From the velocity field contour we observe significant meandering of the turbine wakes in the horizontal plane. At the same time patches of high speed wind can also be seen passing through the spaces between turbine columns. Unlike the flow field, the adjoint field of figure 5(b) evolves backward in time, and propagates in the upstream direction. The adjoint field in general depends strongly upon the definition of the cost functional and its evolution is decided by the development of the flow field. In the current study, the adjoint field has an initial value of $\tilde{\xi}=0$, and it grows gradually backward in time, with the turbines acting as a source. The reason why the adjoint develops from the location of turbines lies in the fact that we evaluate the cost function or power at those turbines (c.f. Eq. 8 and 11). This adjoint field decides the magnitude and direction of the gradient.

Figure 6 shows the behavior of the thrust coefficient and the farm power output for the controlled wind farm. Note that, the nondimensional simulation time $t\left(u_{*} / D\right)=25$ is approximately equivalent to 45 minutes. It is observed in figure 6 (a), that for the time period $(0,1.4), C_{T}^{\prime}$ is constant. Here the optimal control is not yet active. Once the control algorithm is activated, we observe strong changes in $C_{T}^{\prime}$ in response to the turbulent flow field. Note that in the control the lower and upper limit for the $C_{T}^{\prime}$ are set respectively to 0 and 4 using box constraint. This is necessary, because if left entirely to the controller, it may designate a very high or even a negative value to the thrust coefficient, as long as optimized power output is achieved. However, turbines with very large $C_{T}^{\prime}$ may not be feasible, also negative $C_{T}^{\prime}$ means the turbine is pumping energy into the boundary layer and therefore the range for $C_{T}^{\prime}$ is restricted to the more realizable value.

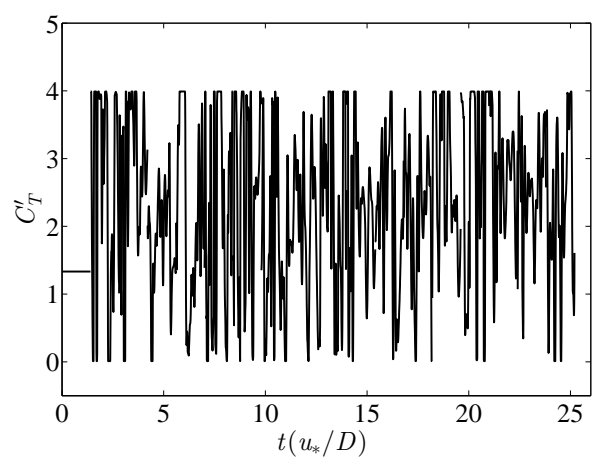

(a)

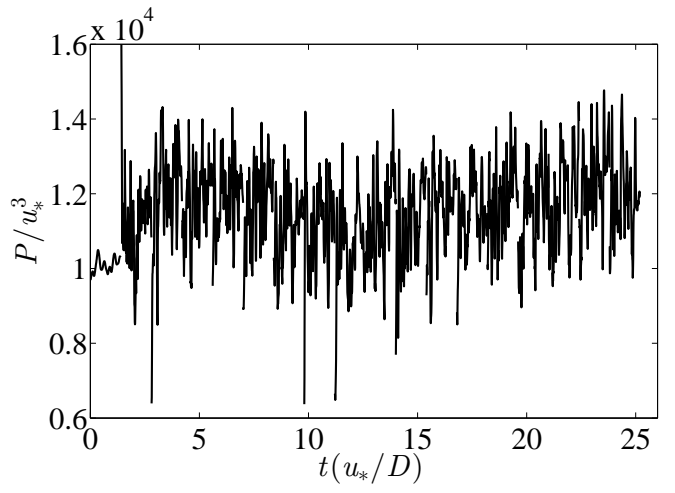

(b)

Figure 6. Time evolution of (a) Thrust coefficient of one of the turbines in the farm, (b) Farm power output. 


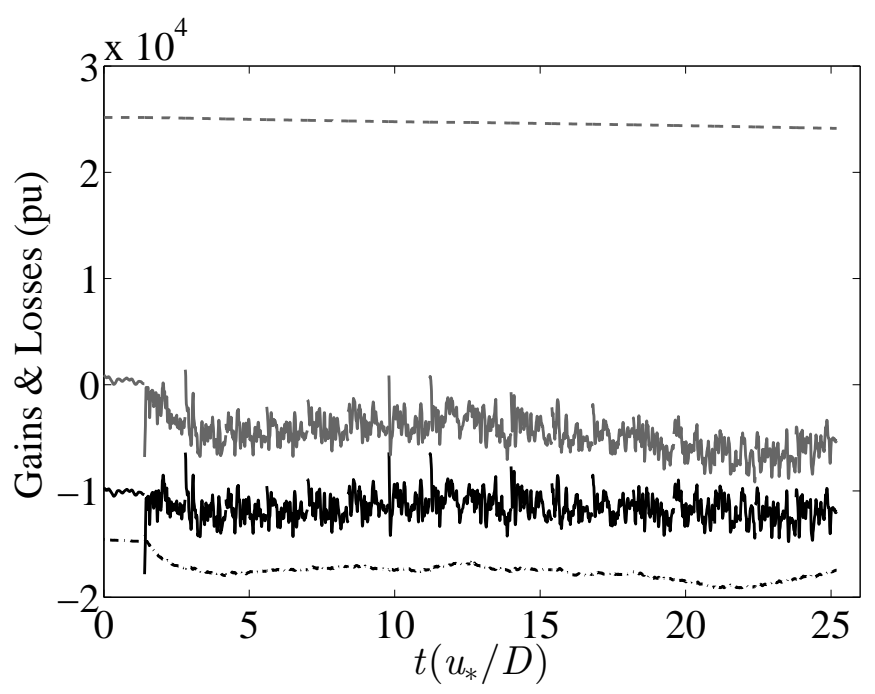

Figure 7. Gains and Losses. Dashed line: driving pressure force; grey line: rate of change of kinetic energy; dark line: farm power; dot dashed line: dissipation.

Figure 6 (b) shows the time series of the total instantaneous power extracted from the farm. It is obvious from the figure that there is an increase in power extraction when the controller is active. This instantaneous power when integrated over the advancement time according to

$$
P_{\text {tot }}=\int_{i T_{\mathrm{A}}}^{(i+1)} \sum_{n=1}^{T_{\mathrm{A}}}-P_{n} d t
$$

where $i=0,1,2 \ldots$, we find that there is $20 \%$ gain in the total power because of optimization. Further evaluation shows that the optimized power is also higher (by approximately 20\%) than the peak power of figure 4 for the uncontrolled wind farm case.

Finally, in figure 7, we evaluate gains and losses terms in the instantaneoous kinetic energy equation. The balance of kinetic energy, obtained from the NS equation Eq. (1), may be written as

$$
\frac{d}{d t} \int_{\Omega} E d \Omega=-\int_{\Omega} \frac{\partial p}{\partial x} \tilde{u}_{1} d \Omega-\sum_{n=1}^{N_{t}} P_{n}-\int_{\Omega} \mathscr{D} d \Omega .
$$

where $E$ is the kinetic energy of the flow and $\mathscr{D}$ is a turbulent dissipation. The external energy input to the boundary layer in the form of pressure gradient (first term on the rhs) remains almost constant. While the optimization increases the extracted power, it is also responsible for the significant increase in the loss to the dissipation (dot dashed line). As a consequence, the flow in the boundary layer slows down resulting in the negative rate of kinetic energy (grey line). It may be conceivable that slowdown of the boundary layer is not a problem, so long as it leads to the gain in the power, but in that case the transient flow condition persists throughout the simulation. To achieve a new regime of steady state during the optimization, it may be necessary to redefine the objective function, e.g. by penalizing the boundary-layer deceleration. Nevertheless, also the transient regime found in the current study may be of interest. In particular, an increased energy extraction (20\%) for a time period of 45 minutes may be relevant for primary grid support.

\section{Conclusion}

In this paper we present the methodology and the implementation of the gradient based optimization technique for the power optimization of a large scale wind farm. Formal derivation of the adjoint equations necessary for gradient calculation has also been presented. Additionally, we have also provided some insight into the adjoint field and its importance in a large scale optimization problem. Optimization is performed in the LES - based receding horizon framework, in which control $C_{T}^{\prime}$ is optimized over the fixed time horizon, before advancing forward to the next iteration. 
Gain in the total power output for the current cost functional definition is about $20 \%$ with respect to the uncontrolled case. However, this increase in the extracted power is related to a deceleration of the boundary layer, at the same time increasing the turbulent dissipation. No new statistical equilibrium was found yet, though also the transient regime identified in the current work, with an increased energy extraction that is sustained for a period of approximately 45 minutes may be interesting from a practical point of view for, e.g., primary grid support. Further research focusses on the identification of a statistical equilibrium and the potential gains in such a regime.

\section{Acknowledgments}

J.M. acknowledges support from the European Research Council (FP7-Ideas, grant no. 306471). Simulations were performed on the computing infrastructure of the VSC Flemish Supercomputer Center, funded by the Hercules Foundation and the Flemish Government.

\section{References}

${ }^{1}$ Calaf, M., Meneveau, C., and Meyers, J., "Large eddy simulation study of fully developed wind-turbine array boundary layers," Phys. Fluids, Vol. 22, 2010, pp. Art no 015110. DOI: 10.1063/1.3291077.

${ }^{2}$ Hansen, K. S., Barthelmie, R. J., Jensen, L. E., and Sommer, A., "The impact of turbulence intensity and atmospheric stability on power deficits due to wind trubine wakes at Horns Rev wind farm." Wind Energy., Vol. 15, 2011, pp. 183-196.

${ }^{3} \mathrm{Lu}, \mathrm{H}$. and Porté-Agel, F., "Large-eddy simulation of a very large wind farm in a stable atmospheric boundary layer." Phys. Fluids, Vol. 23, 2011, pp. 065101.

${ }^{4}$ Newman, B. G., "The Spacing of Wind Turbines in Large Arrays," Energy Conv., Vol. 16, 1976, pp. 169-179.

${ }^{5}$ Meyers, J. and Meneveau, C., "Optimal turbine spacing in fully developed wind-farm boundary layers," Wind Energy, Vol. 15, 2012, pp. 305-317.

${ }^{6}$ Soleimanzadeh, M., Wisniewski, R., and StoyanKanev, "An optimization framework for load and power distribution in wind farms," J. Wind Eng. Ind. Aerodyn., Vol. 107-108, 2012, pp. 256-262.

${ }^{7}$ Fleming, P., Gebraad, P., Lee, S., Churchfield, M., Scholbrock, A., Michalakes, J., Johnson, K., and Moriarty, P., "SOWFA Super-Controller: A High-Fidelity Tool for Evaluating Wind Plant Control Approaches," Proc. EWEA 2013, 4-7 February 2013, Vienna, Austria, Vol. EWEA, 2013, pp. 1-12.

${ }^{8}$ Bewley, T. R., Moin, P., and Temam, R., "DNS-based predictive control of turbulence: an optimal benchmark for feedback algorithms," J. Fluid Mech., Vol. 447, 2001, pp. 179-225.

${ }^{9}$ Meyers, J. and Meneveau, C., "Large eddy simulations of large wind-turbine arrays in the atmospheric boundary layer," 48th AIAA Aerospace Sciences Meeting Including the New Horizons Forum and Aerospace Exposition, Orlando, Florida, Vol. AIAA 2010-827, 2010, pp. 1-10

${ }^{10}$ Moeng, C.-H., "A large-eddy simulation model for the study of planetary boundary-layer turbulence." J. Atmos. Sci., Vol. 6, 1984, pp. 2311.

${ }^{11}$ Pironneau, O., "On optimum design in fluid mechanics," J. Fluid Mech., Vol. 64, 1974, pp. 97-110.

${ }^{12}$ Jameson, A., "Aerodynamic Design via Control Theory," J. Sci. Comput., Vol. 3, 1988, pp. 233-260.

${ }^{13}$ Choi, H., Hinze, M., and Kunisch, K., "Instantaneous control of backward-facing step flows," Applied Numerical Mathematics, Vol. 31, 1999, pp. $133-158$.

${ }^{14}$ Tröltzsch, F., Optimal Control of Partial Differential Equations: Theory, Methods, and Applications, American Mathematical Soc., 2010.

${ }^{15}$ Mason, P. J. and Thomson, D. J., "Stochastic backscatter in large-eddy simulations of boundary layer." J. Fluid Mech., Vol. 242, 1992, pp. 51.

${ }^{16}$ Press, W. H., Teukolsky, S. A., Vetterling, W. T., and Flannery, B. P., Numerical Recipes in FORTRAN77: The art of scientific computing, Cambridge University Press, 2nd edition, 1996.

${ }^{17}$ Delport, S., Baelmans, M., and Meyers, J., "Constrained optimization of turbulent mixing-layer evolution," J. Turbul., Vol. 10, 2009, pp. 18.

${ }^{18}$ Delport, S., Baelmans, M., and Meyers, J., "Maximizing dissipation in a turbulent shear flow by optimal control of its initial state," Phys. Fluids, Vol. 25, 2011, pp. 045105.

${ }^{19}$ Meyers, J. and Meneveau, C., "Flow visualization using momentum and energy transport tubes and applications to turbulent flow in wind farms," Journal of Fluid Mechanics, Vol. 715, 2013, pp. 335-358. 